\title{
¿Es útil la amniotomía de rutina en el trabajo de parto de inicio espontáneo? Una revisión de la literatura
}

\author{
Is routine amniotomy during spontaneous labor useful? Review of the literature
}

\author{
Walter Ventura ${ }^{1}$, Nelly Lam-Figueroa ${ }^{1,2}$ \\ ${ }^{1}$ Unidad de Medicina Fetal, Instituto Nacional Materno Perinatal. Lima, Perú. \\ ${ }^{2}$ Investigador Permanente del Instituto de Investigaciones Clínicas, Facultad de Medicina, Universidad Nacional Mayor de San Marcos. Lima, Perú.
}

\begin{abstract}
Resumen
La amniotomía de rutina o sistemática, practicada en gestantes con trabajo de parto de inicio espontáneo, se da en gran parte de hospitales del Perú y el mundo, documentándose su prevalencia hasta en $77 \%$. Sin embargo, poco se conoce acerca de los beneficios y daños que puede ocasionar en el feto y en la madre. Se realizó una búsqueda de la literatura médica, con la finalidad de encontrar evidencia científica sólida que respalde o rechace esta práctica. Se llega a la conclusión que la amniotomía practicada sistemáticamente altera la evolución fisiológica del trabajo de parto, acortándolo hasta en 50 minutos en promedio; sin embargo, este acortamiento no tiene trascendencia clínica ni para el feto ni para la madre. Por el contrario, su práctica de rutina puede aumentar el número de partos disfuncionales y el puntaje Ápgar menor de siete a los cinco minutos. Palabras clave: Amniotomía; distocia; trabajo de parto.
\end{abstract}

\begin{abstract}
Systematic amniotomy in pregnant women with spontaneous labor is largely practiced in Peruvian and world hospitals with documented prevalence up to $77 \%$. Little is known on benefits and harms resulting to both fetus and mother. We searched the medical literature in order to find solid evidence to support or reject this practice. We conclude that systematic amniotomy alters the physiological evolution of labor shortening it about 50 minutes average. However, this has no clinical significance on both fetus and mother. Conversely, routine practice may increase incidence of dysfunctional labor and five minutes less than seven Apgar score.
\end{abstract}

Key words: Amniotomy; dystocia; labor, obstetric.

\section{Introducción}

La amniotomía es la rotura deliberada o artificial de las membranas corioamnióticas, realizada digitalmente o a través de instrumentos denominados amniótomos, como el de Beacham, o empleando pinzas, como la Kelly o Köecher. Es un procedimiento simple, que ha sido utilizado a través de muchos años en pacientes que no han estado en trabajo de parto, para la inducción del parto, solo o asociado a otros fármacos, como prostaglandinas y oxitocina. También, ha sido utilizada en pleno trabajo de parto, para verificar el color y aspecto del líquido amniótico, y para la colocación de electrodos en el cuero cabelludo fetal, con la finalidad de obtener el registro de la actividad cardiaca del feto o para la obtención de sangre y la medición del pH, en el diagnóstico de acidosis fetal ${ }^{(1,2)}$. Sin embargo, es sabido que el color meconial del líquido amniótico puede no responder a la hipótesis de que el feto está en sufrimiento fetal y se sabe que $20 \%$ de los fetos a término presentan líquido meconial, sin otro hallazgo de distrés fetal ${ }^{(3)}$. Asimismo, ha sido utilizada para aumentar la intensidad y frecuencia de las contracciones uterinas y acortar de esta forma la duración del trabajo de parto ${ }^{(4)}$. Por otro lado, hay que recordar que fisiológicamente $70 \%$ de gestantes llega a dilatación completa con las membranas intactas ${ }^{(5)}$.
En la última década, se ha publicado los informes de varios estudios clínicos aleatorizados que comparan la amniotomía de rutina con un intento de preservar las membranas. Cada uno de estos estudios proporciona información sobre los efectos de la amniotomía en la evolución del trabajo de parto. Sin embargo, debido a los tamaños muestrales restringidos, es limitada la capacidad de estos estudios para abordar los efectos de la amniotomía sobre los indicadores de la morbilidad materna y neonatal ${ }^{(6)}$.

\section{Antecedentes históricos}

Por todo el mundo, existió la idea de que un fragmento de la membrana ovular adosada a la cabeza proporcionaba buena fortuna y buena suerte a quien nacía con ella. En 1799, el precio por el que pagaban los marineros británicos por un pedazo de membrana era 30 guineas; eran los días de las guerras napoleónicas y las grandes batallas de mar ${ }^{(5)}$.

La rotura artificial de membranas es una práctica que los obstetras han usado ampliamente durante las últimas décadas y que hoy se puede decir es uno de los procedimientos obstétricos más utilizados. Se ha propugnado la amniotomía temprana como componente de un manejo activo del trabajo de parto, abordaje propuesto como un recurso posible de reducir la elevada tasa de cesáreas, tanto en América del
Norte como en Europa, especialmente en el Hospital Dublín del Reino Unido, donde se describió por primera vez el término manejo activo del trabajo de parto y la amniotomía como su principal componente ${ }^{(7)}$.

La amniotomía, como técnica obstétrica, fue introducida por primera vez en 1756 , por Thomas Denman. Es en 1928, cuando Kreis propugna la amniotomía, como una técnica que debe practicarse en toda paciente, señalando que las membranas generan contracciones espasmódicas que retrasan el curso normal del parto ${ }^{(5)}$. En 1984, O'Driscoll publicó una extensa experiencia clínica, a la cual llamó manejo activo de trabajo de parto, con la finalidad de reducir la tasa de cesáreas, previniendo las distocias y acortando el trabajo de parto. Este protocolo de manejo consiste en un diagnóstico temprano del trabajo de parto, practicar amniotomía rutinaria en el lapso de una hora de admitida la paciente, y evaluación cada dos horas de la evolución del parto, administrando oxitocina cuando no hay progresión del trabajo de parto. Si bien con este protocolo se ha logrado reducir la tasa de cesáreas, no hay que olvidar que se trata de un trabajo no aleatorizado y realizado solo en nulíparas, sin mayores factores de riesgo, por lo que su recomendación es limitada, debido al bajo nivel de evidencia de este tipo de estudios, que no corresponden a ensayos clínicos ${ }^{(7)}$. 
Casi 50 años después del reconocimiento del rol de las prostaglandinas, tanto para el inicio del parto y la dilatación cervical, existe un sustento fisiológico para los efectos observados en la amniotomía. Las membranas fetales y la decidua uterina producen grandes cantidades de prostaglandina E2 y F2 $\alpha$ y es sabido que la sola manipulación y más aún la rotura de las membranas fetales causan incremento de la síntesis de prostaglandinas ${ }^{(8)}$.

Con la ausencia de la bolsa de las aguas, desaparecería la acción protectora que esta tiene sobre la cabeza del feto. Así, se demostró que la presión registrada a nivel cefálico es mayor que en el resto del cuerpo y que esta es mayor cuando las bolsas están rotas, observándose además mayor deformación de la cabeza fetal ${ }^{(8,9)}$.

La fuerte compresión de la cabeza, producida por las contracciones uterinas, ocasionan estimulación vagal, que se traduce en la presencia de desaceleraciones tempranas o precoces, en el trazado cardiotocográfico intraparto (dip I). Por otro lado, existe asociación de dips I con alteraciones en el electroencefalograma fetal, pero no se sabe si esto produciría daño cerebral permanente. La presencia de dips I es hasta $21 \%$, cuando las membranas se rompen a los 4 a $5 \mathrm{~cm}$ de dilatación y, por el contrario, solo $3 \%$, cuando se mantienen íntegras hasta el final (10-12).

También, se observó que los neonatos que mantuvieron las membranas íntegras hasta el final, tienen un $\mathrm{pH}$ umbilical y una saturación de hemoglobina significativamente más alta y una $\mathrm{pCO}_{2}$ significativamente más baja que los nacidos de partos con membranas rotas precozmente ${ }^{(13)}$.

No existen datos a nivel nacional sobre la frecuencia o prevalencia de la práctica de la amniotomía. Es sabido que se viene practicando casi rutinariamente, en muchas instituciones, como en el Instituto Nacional Materno Perinatal (INMP), con la idea de reducir el tiempo del trabajo de parto. Esta práctica está apoyada en algunas publicaciones de poco rigor metodológico y con resultados contradictorios, sin valorar los otros posibles resultados adversos, como distocia por enlentecimiento o por hiperdinamia, terminando en un mayor número de cesáreas, mayor uso de antibióticos, y otras complicaciones menos frecuentes, como endometritis, infección neonatal, prolapso de cordón, entre otros. Zamudio y col., en un estudio retrospectivo en el Hospital Militar Central, en la búsqueda de factores asociados al prolapso de cordón, encontró que 30\% de estos estuvo asociado a amniotomía intrapar- to ${ }^{(14)}$. Mato y col. comunicó recientemente un caso de embolismo de líquido amniótico asociado a amniotomía ${ }^{(15)}$.

Cuellar y col, en un estudio no publicado, presentado en un congreso brasileño, encontró que, en su hospital, 69,8\% había tenido amniotomía y que esta se había realizado en las primeras dos horas luego del internamiento ${ }^{(16)}$. Schwarcz encuentra una prevalencia de hasta $75 \%{ }^{(5)}$.

Con la finalidad de responder a la pregunta de si es beneficiosa la amniotomía practicada de rutina en el trabajo de parto, se realizó una revisión bibliográfica en las bases de datos Medline, Embase y Lilacs, utilizando los términos amniotomy, amniotomía, amniorrexis, artificial rupture of the amniotic membranes; se cruzó con labor y se eliminó la palabra induction, encontrándose 74 citas publicadas en los últimos 25 años, desde artículos de reporte de casos hasta 2 revisiones sistemáticas de la biblioteca Cochrane, teniendo como resultado principal el acortamiento del trabajo de parto, una de Fraser y col, actualizada en 1999, y la otra de Smyth y col, actualizada hasta $2007^{(1,6)}$.

\section{Resultados}

El de Fraser y col. ${ }^{(2)}$ es uno de los más importantes ensayos clínicos aleatorizados, Canadian early amniotomy study, en el que demuestran que la amniotomía disminuye el tiempo promedio del trabajo de parto, en 136 minutos, pero solo cuando la dilatación es mayor de $3 \mathrm{~cm}$, no encontrando diferencia cuando la dilatación es menor de $3 \mathrm{~cm}$. Sin embargo, en los resultados trascendentales (end points primarios) -como son número de cesáreas y puntuación Ápgar baja- no hubo diferencia, por lo que deberíamos preguntarnos si existe verdaderamente algún beneficio clínico trascendente al acortar el trabajo de parto. Igualmente Lopez-Zeno y col. ${ }^{(17)}$ no demuestran algún efecto en la tasa de cesáreas. D'orsi y col. ${ }^{(18)}$ encuentran que la amniotomía, por el contrario, es un factor protector de cesárea, mediante un análisis de regresión.

N. Mikki y col. ${ }^{(19)}$, en un estudio clínico aleatorizado llevado a cabo en un hospital palestino, de enseñanza como el nuestro, agrupó a las gestantes de riesgo bajo en nulíparas y multíparas y encontró que, en los que se realizó amniotomía intraparto de rutina hubo una reducción significativa en el acortamiento del trabajo de parto, menor uso de oxitocina y ninguna diferencia en el modo del parto. Recomienda sin embargo que deba reservarse este uso para trabajos de parto que no progresan adecuadamente.
Kathleen $\mathrm{H}$ y col. ${ }^{(20)}$ no encuentran diferencias significativas en la duración del trabajo de parto y, por el contrario, hallan mayor número de neonatos con caput succedaneum, distorsión de los huesos de la calota, desaceleraciones tipo dip I y mayor disminución del pH, medido en cordón umbilical, recomendando su uso solo en situaciones especiales.

Johnson N y col. ${ }^{(21)}$, en un ensayo clínico que logró reunir a 1540 mujeres, demuestran que la amniotomía rutinaria acorta el trabajo de parto solo en mujeres nulíparas y no en mujeres multíparas y no encuentra diferencia en la tasa de cesáreas.

Fraser y col. ${ }^{(1)}$, en un metaanálisis para la librería Cochrane, en la que solo se incluye dos ensayos, desechándose varios, por la inadecuada calidad metodológica, encontraron que la amniotomía estuvo asociada con una reducción de la duración del trabajo de parto, entre 60 y 120 minutos. Hubo una tendencia marcada hacia un aumento en el riesgo de parto por cesárea (OR: 1,26; IC 95\%: 0,96 a 1,66). El grupo con amniotomía temprana tuvo menor puntaje Ápgar (menor de 7 a los 5 minutos) (OR: 0,54; IC 95\%: 0,30 a 0,96). Los grupos fueron similares con respecto a otros indicadores del estado neonatal $(\mathrm{pH}$ arterial del cordón, ingresos a la unidad de cuidados intensivos neonatales). También, encontraron que el grupo de amniotomía tuvo menor necesidad de usar oxitocina (OR: 0,79; IC 95\%: 0,67 a 0,92).

Smith y col. ${ }^{(6)}$, en la última revisión sistemática para la librería Cochrane, del 2007, con un total de 4893 mujeres, concluyen que no existe diferencia significativa en el acortamiento del trabajo de parto, incidencia de cesáreas, morbilidad neonatalmaterna y entre otras variables secundarias. Solo se demuestra que existen menos partos distócicos en el grupo de amniotomía, basado en el resultado de solo dos estudios. Si bien ellos reúnen catorce estudios clínicos, los criterios de inclusión y la metodología de análisis son muy heterogéneos, por lo que recomiendan realizar ensayos clínicos aleatorizados multicéntricos, con variables finales de trascendencia clínica.

En pocos estudios se evaluó la satisfacción materna con la práctica de la amniotomía y, en los que fue evaluada, se muestra mayor disconfort e inseguridad con el trabajo de parto, por parte de las pacientes ${ }^{(22)}$. Los médicos no debemos asumir que el acortar un trabajo de parto esperado por una mujer sea lo que verdaderamente ella desee. Así, se ha visto que algunas mujeres 
sienten perder el control del parto cuando ellas notan que han roto las membranas y se angustian mucho más ${ }^{(22)}$. Algunas piensan que han perdido la protección de la cabeza del bebé y se genera dudas y disconfort durante todo el trabajo de parto ${ }^{(22)}$.

La tendencia hacia un incremento en el índice de cesáreas observada en el metaanálisis, combinada con la evidencia de un aumento en la tasa horaria de anomalías en el monitoreo cardíaco fetal y con el aumento en la frecuencia de cesáreas por sufrimiento fetal, observada en un estudio clínico multicéntrico (Goffinet, 1997) ${ }^{(23)}$ sugieren que deberíamos atenuar nuestro entusiasmo por la implementación de una política de amniotomía temprana de rutina, como una intervención aislada. Los efectos adversos de la amniotomía (sobre el trazado de la frecuencia cardiaca fetal y, consecuentemente, sobre el riesgo de cesárea por sufrimiento fetal) pueden ser mucho más importantes en los centros donde el monitoreo electrónico fetal se usa habitualmente sin muestreo de sangre fetal como un complemento. Estos efectos se verían probablemente atenuados por muestra de sangre fetal (Thacker, 1999) ${ }^{(24)}$, por amnioinfusión en caso de desaceleraciones variables preocupantes (Hofmeyr, 1999) ${ }^{(25)}$ o por una combinación de ambas técnicas. En esencia, esto implica brindar intervenciones para minimizar los efectos secundarios de una intervención de rutina anterior. Dados los conocimientos actuales, parecería ser que, reservar la amniotomía para trabajos de parto con una evolución lenta es una conducta razonable.

Los ensayos clínicos aleatorizados más grandes, como el UK Amniotomy Group (1994) ${ }^{(26)}$, el Canadian Early Amniotomy Study Group (1992) (2) y el estudio colaborativo multicéntrico en América Latina y el Caribe, por Schwarcz (1975) ${ }^{(5)}$, en la que participó nuestro país, con hospitales como el INMP, el Hospital San Bartolomé y hospitales de varias provincias, nos dicen que, al igual que sucede con la episiotomía de rutina, la amniotomía practicada sin indicación debe quedar como una práctica obstétrica, sepultada por la medicina basada en evidencias, y deberá ser nuestro juicio clínico según el escenario al que nos enfrentemos el que decida si debemos disponer de ella o no.

En conclusión, no se ha demostrado que el acortamiento del trabajo de parto tenga un resultado clínicamente trascendente en la madre o el feto. Las evidencias actuales no permiten hacer una recomendación generalizada acerca de la amniotomía rutinaria. No existe un nivel de evidencia significativo que sustente la reducción en el número de cesáreas; por el contrario, existen observaciones que demuestran el aumento en el mismo. Queda por determinar la efectividad de la amniotomía sola como manejo del parto que ha iniciado espontáneamente y que se ha prolongado.

Como recomendaciones y en base al nivel de evidencia encontrado en la literatura recomendamos el uso restringido de la amniotomía en el trabajo de parto de inicio espontáneo, salvo en casos justificados clínicamente.

\section{REFERENCIAS BIBLIOGRÁFICAS}

1. Fraser WD, Turcot L, Krauss I, Brisson-Carrol G. Amniotomy for shortening spontaneous labour. Cochrane Database Syst Rev. 2000(2): CD000015.

2. Fraser WD, Marcoux S, Moutquin JM, Christen A. Effect of early amniotomy on the risk of dystocia in nulliparous women. N Engl J Med. 1993;328:11459.

3. Gibb D, Arulkumaran S. Cardiotocograph interpretation: clinical scenarios. Meconiumstained amniotic fluid. En: Gibb D, Arulkumaran $S$ (eds). Fetal monitoring in practice. Oxford: Butterworth-Heinemann; 1992. p. 130.

4. Neilson JP, Comments: Amniotomy for shortening spontaneous labour. REVISTA. 2008;111(1):2045.

5. Schwarcz R, Balizan JM, Nieto F, Tenzer SM. La rotura precoz de las membranas ovulares y sus efectos sobre el parto y el neonato. Investigación colaborativa entre maternidades de América Latina. Montevideo: Centro Latinoamericano de Perinatología y de Desarrollo Humano-OPS; 1975.

6. Smyth RM, Alldred SK, Markham C. Amniotomy for shortening spontaneous labour. Cochrane Database Syst Rev. 2007(4):CD006167.

7. O'Driscoll K, Foley M, MacDonald D. Active management of labor as an alternative to cesarean section for dystocia. Obstet Gynecol. 1984:63:485-90.

8. Stewart P, Kennedy JH, Calder AA. Spontaneous labour: when should the membranes be ruptured? Br J Obstet Gynaecol. 1982;89:39-43.

9. Ingelman-Sundberg A, Lindgren L. Intrauterine measurement of pressure during labor. J Obstet Gyanaec Br Emp. 1955;62:629.

10. Schwarcz R, Strada Saenz E, Althabe 0, Fernandez Funes J, Caldeyrobarcia R. Pressure exerted byuterine contractions on the head ofth-s human fetus during labor. In: Perinatal Factors Affecting Human Development. Washington D.C.: PAHO; 1969. p. 115.

11. Garcia AE. Effects of uterine contractions on the EEG of the human fetus during labor. In: Perinatal Factors affecting Human Development. Washington D.C.: PAHO; 1969. p. 127.

12. Schwarcz R, Althabe 0, Belitzky R, Lanchares JL, Alvarez $\mathrm{R}$, Berdaguer $\mathrm{P}$, et al. Fetal heart rate patterns in labors with intact and with ruptured membranes. J Perinat Med. 1973;1:153.

13. Schwarcz R, Martell M, Belizan JM, Nieto F. El equilibrio ácido base de la sangre del cordón umbilical en partos con rotura precoz y tardía de las membranas. VI reunión de la Asociación Latinoamericana de Investigación en Reproducción Humana. Lima, Perú. 1974.

14.Zamudio AE. Prolapso de cordón umbilical. Hospital Central No 1, 1966 -1973. Tesis para obtener el grado de bachiller. Facultad de Medicina, UNMSM. 1974.

15. Mato J. Suspected amniotic fluid embolism following amniotomy: a case report. AANA J. 2008;76(1):53-9.

16. Cuellar J. La ruptura artificial de las membranas y su relación con la evolución del parto. Jornada Regional de Residencia Médica. Santa Cruz de la Sierra: Secretaria Regional de Salud; 1994. p. 189-208.

17. Lopez-Zeno JA, Peaceman AM, Adashek JA, Socol ML. A controlled trial of a program for the active management of labor. $\mathrm{N}$ Engl J Med. 1992;326:450-4.

18. D'Orsi E, Chor D, Giffin K, Angulo-Tuesta A, Barbosa GP, Gama Ade S, Reis AC. Factors associated with cesarean sections in a public hospital in Rio de Janeiro, Brazil. Cad Saude Publica. 2006;22(10):2067-78.

19. Mikki N, Wick L. A trial of amniotomy in a Palestinan Hospital. J Obstet Gynaecol. 2007;27(4):368-73.

20. Lynaugh $\mathrm{KH}$. The effects of early elective amniotomy on the length of labor and the condition of the fetus. J Nurse-Midwifery. 1980;25(4):3-9.

21. Johnson N, Lilford R. Randomized trial comparing a policy of early with selective amniotomy in uncomplicated labour at term. $\mathrm{Br} J$ Obstet Gynecol. 1997;104(3):340-6.

22. Robinson J. Her master's voice? Amniotomy in Dublin. Brit J Midwifery. 2000;8:110.

23. Goffinet F, Fraser W, Marcoux S, Bréart G, Moutquin JM, Daris M. Early amniotomy increases the frequency of fetal heart rate abnormalities. $\mathrm{Br} J$ Obstet Gynaecol. 1997;104(5):632-40.

24. Thacker SB, Stroup D, Chang M. Continuous electronic heart rate monitoring for fetal assessment during labor. Cochrane Database Syst Rev. 2001;(2):CD000063.

25. Hofmeyr GJ. Amnioinfusión para la ruptura de las membranas antes de término. Cochrane. 1999;(4). Oxford.

26. The UK Amniotomy Group. A multicentre randomized trial of amniotomy in spontaneous first labour at term. Br J Obstet Gynaecol. 1994;101(4):307-9.

Manuscrito recibido el 21 de abril de 2008 y aceptado para publicación el 3 de junio de 2008.

Correspondencia:

Dr. Walter R. Ventura Laveriano

Unidad de Medicina Fetal

Instituto Nacional Materno Perinatal

Correo-e: walterichard@hotmail.com nlamfigueroa@yahoo.es 